\title{
Chemostat Culture for Yeast Physiology and Experimental Evolution
}

\author{
Maitreya J. Dunham, ${ }^{1}$ Emily O. Kerr, Aaron W. Miller, and Celia Payen \\ Department of Genome Sciences, University of Washington, Seattle, Washington 98195
}

\begin{abstract}
Continuous culture provides many benefits over the classical batch style of growing yeast cells. Steadystate cultures allow for precise control of growth rate and environment. Cultures can be propagated for weeks or months in these controlled environments, which is important for the study of experimental evolution. Despite these advantages, chemostats have not become a highly used system, in large part because of their historical impracticalities, including low throughput, large footprint, systematic complexity, commercial unavailability, high cost, and insufficient protocol availability. However, we have developed methods for building a relatively simple, low-cost, small footprint array of chemostats that can be run in multiples of 32. This "ministat array" can be applied to problems in yeast physiology and experimental evolution.
\end{abstract}

The most common method of growing yeast cell cultures is the standard "batch" culture. In this regime, a small number of cells are inoculated into nutrient-rich medium and allowed to divide at maximal growth rate through nutrient exhaustion and into saturation. Sampling of the culture for the experiment of choice is most typically done from the saturated culture or from cells growing in mid$\log$ phase before the diauxic shift. Batch culture provides a number of obvious benefits-it is easy, uses glassware commonly available in any laboratory, and is consistent with a vast literature. However, batch cultures can be problematic for certain applications. When comparing cells of different genotypes, for example, differences in the maximal growth rate are a common phenotype. Many other phenotypes co-vary with growth rate, leading to a nonspecific suite of cell biological, gene expression, and other physiological changes that may not be directly related to the mutation of interest (Regenberg et al. 2006; Castrillo et al. 2007; Brauer et al. 2008).

Batch culture has also been a standard for long-term evolution experiments. Depending on the question being asked, batch culture with serial dilution can be a simple and scalable solution (e.g., Zeyl et al. 2003; Lang et al. 2011). However, such cultures are only rarely propagated in a relatively constant environment and growth rate regime because doing so can require a heroic sampling regimen where back dilution is performed multiple times per day (e.g., Torres et al. 2010). More typically, cultures are allowed to exhaust nutrients before transfer to new medium, leading to a variation in growth rate and nutrient access over the evolutionary time course. These discontinuities in selective pressure can lead to complex subpopulation structures in which different genotypes specialize for the various stages of the growth cycle (e.g., dividing a few extra times or failing to die after saturation, shortening lag phase,

\footnotetext{
${ }^{1}$ Correspondence: maitreya@uw.edu

(C) 2017 Cold Spring Harbor Laboratory Press

Cite this introduction as Cold Spring Harb Protoc; doi:10.1101/pdb.top077610
} 
or increasing maximal growth rate, all of which have been observed in bacterial serial-dilution-based evolution experiments [Lenski et al. 1998; Rozen and Lenski 2000]).

\section{CONTINUOUS CULTURE}

Cell physiology and experimental evolution can be studied in a more controlled manner using continuous cultures. Continuous culture refers to the utilization of a class of growth apparatus that maintains a constant environment in one of several dimensions, including chemostats (constant growth rate), turbidostats (constant turbidity), and other devices that feed back on $\mathrm{pH}$, oxygen tension, or other parameters. For the purposes of this introduction, we will focus on chemostats. In chemostat culture, cells are grown at a set dilution rate to a nutrient-limited steady state, at which all inputs equal all outputs. This steady state is highly desirable for modeling applications (e.g., Knijnenburg et al. 2009; Reznik et al. 2013) and for perturbation experiments (e.g., Ronen 2006; McIsaac et al. 2012). The precise control over growth rate also allows matching of growth rates between wild-type cultures and more slowly growing mutants, or among diverse strain backgrounds, by forcing the cells to grow at a steady state that is below the maximum growth rate of both (e.g., Hayes et al. 2002; Torres et al. 2007; Skelly et al. 2013). Even small differences in competitive fitness can be accurately measured using the chemostat, making it a useful tool for characterizing individual mutants (Baganz et al. 1998) and collections of strains (Delneri et al. 2008).

Steady state is typically achieved within the first several volume replacements after inoculation of the chemostat and onset of media flow. Steady state can be maintained for up to at least 35 generations before being detectably perturbed by the inevitable influx of de novo mutations and subsequent selection, turning a physiology experiment into an evolutionary one. For evolution experiments, the advantages are continuous growth in a stable environment. This assumption is never strictly true, however, as cultures can change in density and media composition as their genotype composition shifts. Despite this complexity, the primary selection pressure is largely maintained by the nutrient limitation, which is selected by the experimenter.

\section{CHEMOSTAT DESIGN}

The basic plumbing of all chemostat platforms is generally the same (see Fig. 1). Medium is added at a defined rate while spent medium, including cells, overflows the culture at that same rate. Aeration, if desired, is provided by vigorous stirring and/or bubbling of gas through the culture. The media feed,

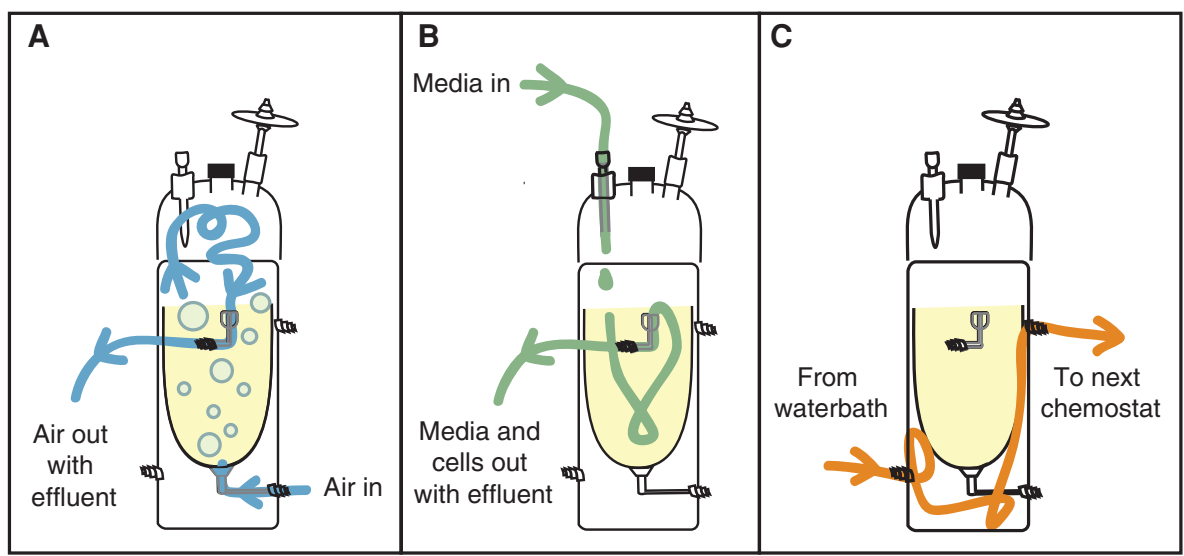

FIGURE 1. Diagram illustrating a basic chemostat. This example of a glass-blown chemostat provides $(A)$ mixing by vigorous aeration, $(B)$ a constant influx of media and overflow of culture effluent, and $(C)$ temperature control via a water jacket. 
M.J. Dunham et al.

aeration, and mixing must be optimized to distribute cells and media components uniformly in both space and time. Chemostat platforms can vary in scale over several orders of magnitude with respect to cell population and working volume (a table of vendors and plans can be found in Dunham [2010]). Commercial fermenters that have been modified for chemostat use, such as those from New Brunswick or Infors, typically range from $50 \mathrm{~mL}$ to $2 \mathrm{~L}$. Custom glass-blown or otherwise home-built devices typically fall toward the lower end of this range and below (e.g., Klein et al. 2013). Microfluidic implementations weigh in at just a few microliters (Cookson et al. 2005; Groisman et al. 2005; Dénervaud et al. 2013). There are several important considerations to take into account when determining which system scale is most appropriate. Media usage in large fermenters may be prohibitively expensive for long-running experiments and many devices would consume a large laboratory space. However, such a large-scale system might be required to maintain adequate representation of highly complex libraries. At the other extreme, although microfluidic devices help address problems with media consumption and parallelization, population sizes may be insufficient for most evolutionary applications. Run times in microfluidic devices are also most compatible with shorter term physiology and perturbation experiments, although this has been improving (Jakiela et al. 2013).

We have sought a compromise solution to these considerations by using a mid-range $20 \mathrm{~mL}$ working volume that can be multiplexed to 32 units in a single array (Miller et al. 2013). For a yeast culture growing at a typical working density, this represents a population size on the order of $10^{8}-10^{9}$ cells. We most typically grow the cultures at a dilution rate of $0.17 \mathrm{vol} / \mathrm{h}$, a rate chosen to sit comfortably below the critical dilution rate at which most laboratory strains switch from mixed respiro-fermentative growth to primarily fermentation when grown in glucose limitation (van Dijken et al. 2000) and comfortably above the point where synchronized metabolic cycling has most commonly been observed ( $\mathrm{Tu} 2005$ ). This dilution rate is also convenient for collecting $1 \mathrm{~mL}$ of culture in 15-20 min for practical passive sampling from the overflow port. One 32-chemostat setup costs approximately $\$ 10,000$ and fits on a benchtop or in a vertical rack configuration. Although the system is still complex with many parts, it can be operated by appropriately trained and supervised undergraduate researchers.

Our setup does have several limitations in its current implementation. First, all 32 chemostats must be operated off the same peristaltic pump. Running a subset of vessels at different dilution rates can be achieved by changing their working volume, or by using additional pumps that run independently. The off-the-shelf construction also lacks many of the sensors that commercial fermenters are equipped with. New advances in miniaturized sensor technology will hopefully address this limitation. Finally, we have not solved the long-appreciated, platform-independent problems of wall growth and media line colonization. Media lines could conceivably be outfitted with an additional air gap to try to limit this problem, although colonization could still exist at the site of entry. We are currently exploring strain-engineering approaches to abrogate this problem.

Despite these technical issues, the chemostat design presented in Protocol: Assembly of a MiniChemostat Array (Miller et al. 2015) has been successful in hundreds of experiments lasting an aggregated sum of tens of thousands of generations. We previously showed its equivalence to commercial chemostat platforms with respect to physiology and experimental evolution outcomes (Miller et al. 2013). We hope that our basic chemostat model and detailed protocols for applying the ministat array to problems in yeast physiology (see Protocol: Chemostat Culture for Yeast Physiology [Kerr and Dunham 2015]) and experimental evolution (see Protocol: Chemostat Culture for Yeast Experimental Evolution [Payen and Dunham 2015]) will encourage additional laboratories to adopt this useful culturing technology for their own applications. These protocols have been optimized to allow utilization of undergraduate researchers working as part of a research team.

This work was supported by grants from the National Institute of General Medical Sciences (P41 GM103533 and R01 GM094306) from the National Institutes of Health and by National Science 
Foundation grant 1120425. M.J.D. is a Rita Allen Foundation Scholar and a Senior Fellow in the Genetic Networks program at the Canadian Institute for Advanced Research. A.W.M. was supported in part by National Institutes of Health (T32 HG00035).

\section{REFERENCES}

Baganz F, Hayes A, Farquhar R, Butler PR, Gardner DCJ, Oliver SG. 1998 Quantitative analysis of yeast gene function using competition experiments in continuous culture. Yeast 14: 1417-1427.

Brauer MJ, Huttenhower C, Airoldi EM, Rosenstein R, Matese JC, Gresham D, Boer VM, Troyanskaya OG, Botstein D. 2008. Coordination of growth rate, cell cycle, stress response, and metabolic activity in yeast. Mol Biol Cell 19: 352-367.

Castrillo JI, Zeef LA, Hoyle DC, Zhang N, Hayes A, Gardner DCJ, Cornell MJ, Petty J, Hakes L, Wardleworth L, et al. 2007. Growth control of the eukaryote cell: A systems biology study in yeast. J Biol 6: 4 .

Cookson S, Ostroff N, Pang WL, Volfson D, Hasty J. 2005. Monitoring dynamics of single-cell gene expression over multiple cell cycles. Mol Syst Biol 1: 2005.0024.

Delneri D, Hoyle DC, Gkargkas K, Cross EJM, Rash B, Zeef L, Leong H-S, Davey HM, Hayes A, Kell DB, et al. 2008. Identification and characterization of high-flux-control genes of yeast through competition analyses in continuous cultures. Nat Genet 40: 113-117.

Dénervaud N, Becker J, Delgado-Gonzalo R, Damay P, Rajkumar AS, Unser M, Shore D, Naef F, Maerkl SJ. 2013. A chemostat array enables the spatio-temporal analysis of the yeast proteome. Proc Nat Acad Sci 110: 15842-15847.

Dunham MJ. 2010. Experimental evolution in yeast: A practical guide. Methods Enzymol 470: 487-507.

Groisman A, Lobo C, Cho H, Campbell JK, Dufour YS, Stevens AM, Levchenko A. 2005. A microfluidic chemostat for experiments with bacterial and yeast cells. Nat Methods 2: 685-689.

Hayes A, Zhang N, Wu J, Butler PR, Hauser NC, Hoheisel JD, Lim FL, Sharrocks AD, Oliver SG. 2002. Hybridization array technology coupled with chemostat culture: Tools to interrogate gene expression in Saccharomyces cerevisiae. Methods 26: 281-290.

Jakiela S, Kaminski TS, Cybulski O, Weibel DB, Garstecki P. 2013. Bacterial growth and adaptation in microdroplet chemostats. Angew Chem Int Ed Engl 52: 8908-8911.

Kerr EO, Dunham MJ. 2015. Chemostat culture for yeast physiology. Cold Spring Harb Protoc doi: 10.1101/pdb.prot089003.

Klein T, Schneider K, Heinzle E. 2013. A system of miniaturized stirred bioreactors for parallel continuous cultivation of yeast with online measurement of dissolved oxygen and off-gas. Biotechnol Bioeng 110: 535-542.

Knijnenburg TA, Daran J-MG, van den Broek MA, Daran-Lapujade PA, de Winde JH, Pronk JT, Reinders MJT, Wessels LFA. 2009. Combinatorial effects of environmental parameters on transcriptional regulation in Saccharomyces cerevisiae: A quantitative analysis of a compendium of chemostat-based transcriptome data. BMC Genomics 10: 53.
Lang GI, Botstein D, Desai MM. 2011. Genetic variation and the fate of beneficial mutations in asexual populations. Genetics 188: 647-661.

Lenski RE, Mongold JA, Sniegowski PD, Travisano M, Vasi F, Gerrish PJ, Schmidt TM. 1998. Evolution of competitive fitness in experimental populations of E. coli: What makes one genotype a better competitor than another? Antonie Van Leeuwenhoek 73: 35-47.

McIsaac RS, Petti AA, Bussemaker HJ, Botstein D. 2012. Perturbation-based analysis and modeling of combinatorial regulation in the yeast sulfur assimilation pathway. Mol Biol Cell 23: 2993-3007.

Miller AW, Befort C, Kerr EO, Dunham MJ. 2013. Design and use of multiplexed chemostat arrays. J Vis Exp e50262.

Miller AW, Kerr EO, Dunham MJ. 2015. Assembly of a mini-chemostat array. Cold Spring Harb Protoc doi: 10.1101/pdb.prot088997.

Payen C, Dunham MJ. 2015. Chemostat culture for yeast experimental evolution. Cold Spring Harb Protoc doi: 10.1101/pdb.prot089011.

Regenberg B, Grotkjaer T, Winther O, Fausbøll A, Akesson M, Bro C, Hansen LK, Brunak S, Nielsen J. 2006. Growth-rate regulated genes have profound impact on interpretation of transcriptome profiling in Saccharomyces cerevisiae. Genome Biol 7: R107.

Reznik E, Mehta P, Segrè D. 2013. Flux imbalance analysis and the sensitivity of cellular growth to changes in metabolite pools. PLoS Comput Biol 9: e1003195.

Ronen M. 2006. Transcriptional response of steady-state yeast cultures to transient perturbations in carbon source. Proc Nat Acad Sci 103:389-394.

Rozen D, Lenski R. 2000. Long-term experimental evolution in Escherichia coli. VIII. Dynamics of a balanced polymorphism. Am Nat 155: 24-35.

Skelly DA, Merrihew GE, Riffle M, Connelly CF, Kerr EO, Johansson M, Jaschob D, Graczyk B, Shulman NJ, Wakefield J, et al. 2013. Integrative phenomics reveals insight into the structure of phenotypic diversity in budding yeast. Genome Res 23: 1496-1504.

Torres EM, Sokolsky T, Tucker CM, Chan LY, Boselli M, Dunham MJ, Amon A. 2007. Effects of aneuploidy on cellular physiology and cell division in haploid yeast. Science 317: 916-924.

Torres EM, Dephoure N, Panneerselvam A, Tucker CM, Whittaker CA, Gygi SP, Dunham MJ, Amon A. 2010. Identification of aneuploidy-tolerating mutations. Cell 143: 71-83.

Tu BP. 2005. Logic of the yeast metabolic cycle: Temporal compartmentalization of cellular processes. Science 310: 1152-1158.

van Dijken JP, Bauer J, Brambilla L, Duboc P, Francois JM, Gancedo C, Giuseppin MLF, Heijnen JJ, Hoare M, Lange HC, et al. 2000. An interlaboratory comparison of physiological and genetic properties of four Saccharomyces cerevisiae strains. Enzyme Microb Technol 26: 706-714.

Zeyl C, Vanderford T, Carter M. 2003. An evolutionary advantage of haploidy in large yeast populations. Science 299: 555-558. 


\section{Chemostat Culture for Yeast Physiology and Experimental Evolution}

Maitreya J. Dunham, Emily O. Kerr, Aaron W. Miller and Celia Payen

Cold Spring Harb Protoc; doi: 10.1101/pdb.top077610

\begin{tabular}{rc}
$\begin{array}{r}\text { Email Alerting } \\
\text { Service }\end{array}$ & Receive free email alerts when new articles cite this article - click here. \\
\hline $\begin{array}{c}\text { Subject } \\
\text { Categories }\end{array}$ & $\begin{array}{c}\text { Browse articles on similar topics from Cold Spring Harbor Protocols. } \\
\text { Cell Culture (301 articles) } \\
\text { Yeast (288 articles) }\end{array}$ \\
\hline
\end{tabular}

